\title{
Lithotripsy and ultrasound: Useful armamentarium in the case of ostial calcified stenosis of the right coronary artery
}

\author{
Lukasz Kozinski, Zbigniew Orzalkiewicz \\ Department of Cardiology, District Hospital, Chojnice, Poland
}

An 88-year-old female was admitted for angioplasty of the right coronary artery (RCA) (Fig. 1A). Two months prior an unsuccessful angioplasty of the RCA was undertaken - a $3.0 \times 20 \mathrm{~mm}$ noncompliant (NC) balloon was unable to dilate ostial stenosis and ruptured during inflation. Intravascular ultrasound (IVUS) showed long, diffuse and a heavily calcified narrowing with two concentric calcified plaques in the ostial and medial portion of RCA with a minimal lumen area (MLA) of $1.7 \mathrm{~mm}^{2}$ and $2.1 \mathrm{~mm}^{2}$, respectively (Fig. 1D1-F1, Suppl. Video 1). The shockwave balloon $(3.0 \times 12 \mathrm{~mm})$ was delivered, several applications had been performed throughout the RCA and finally the shockwave balloon was fully opened (Fig. 1B). The second IVUS run showed multiple cracks in calcified plaques with new acoustic shadowings, especially in a calcified ring of the medial RCA (Fig. 1D2-F2, Suppl. Video 1). Lithotripsy was followed by dilatation with $\mathrm{NC}$ balloon in the ostium of the RCA. Then two drugs eluting stents were implanted.
Post-dilatation with an $\mathrm{NC}$ balloon was performed and consequently the angiographic result was good (Fig. 1C). The final IVUS demonstrated a complete stent apposition and acceptable stent expansion in the ostium of RCA with MLA of $12.0 \mathrm{~mm}^{2}$ (Fig. 1D3-F3, Suppl. Video 1).

Coronary lithotripsy is a novel method for calcified lesion modification, i.e. pulsatile sonic pressure waves generate cracks in both intimal and medial part of the artery wall and enable vessel compliance. Compared with rotablation, this tool is easier to apply and causes fewer complications, e.g. coronary perforation and slow/no-flow phenomenon. Shockwave balloon allows simultaneous guidewire placement during bifurcation intervention. The limitation of this technique is a relatively bulky device therefore some lesions are uncrossable.

Herein, is described the use of lithotripsy for treatment of heavily calcified and undilatable ostial RCA stenosis with a satisfactory procedural result.

Conflict of interest: None declared

Address for correspondence: Lukasz Kozinski, MD, Department of Cardiology, District Hospital, ul. Leśna 10, 89-600 Chojnice, Poland, tel/fax: +48 52 3956639, e-mail: lukekozinski@gmail.com

Received: 18.07.2019 Accepted: 1.01.2020 

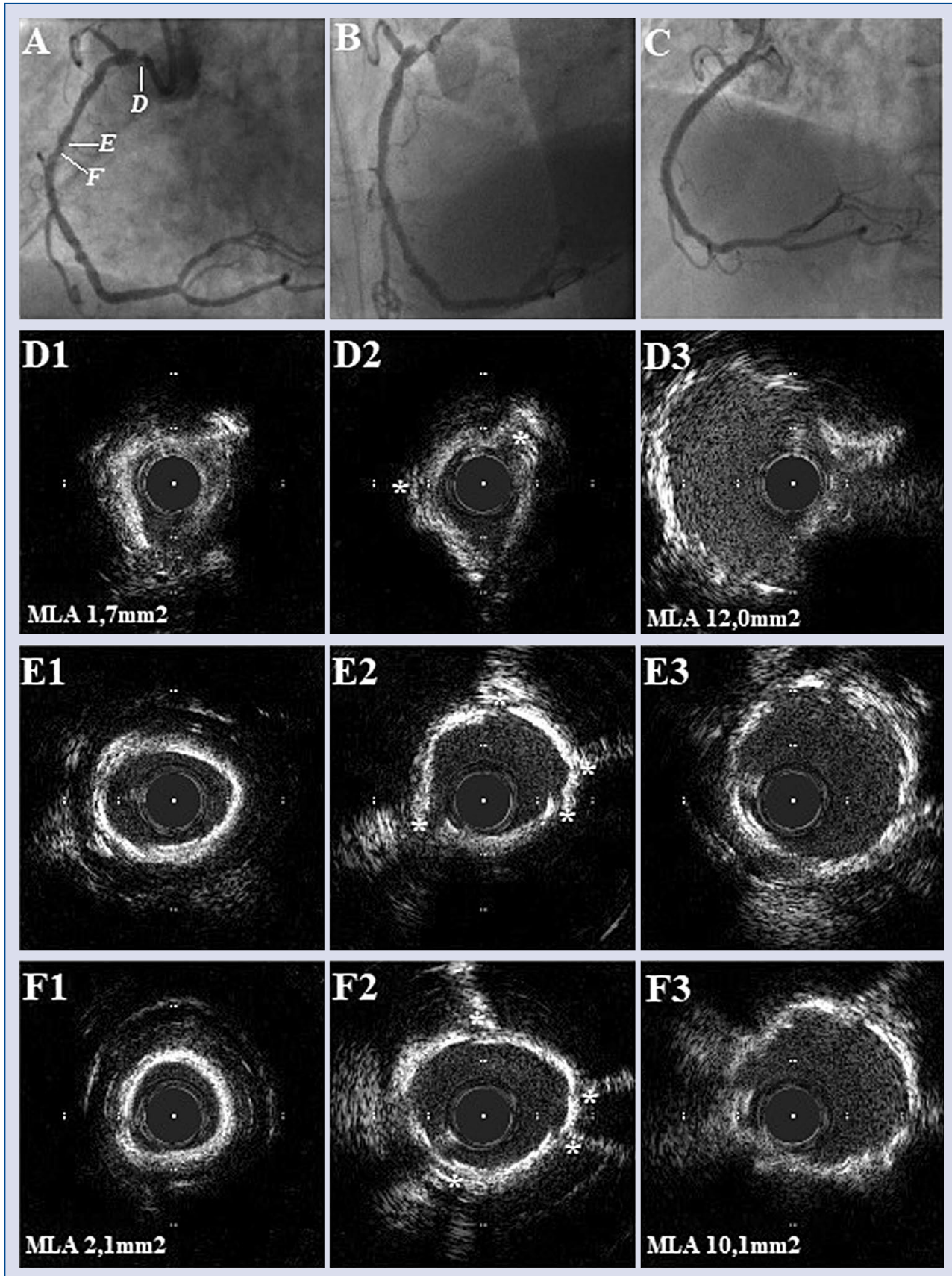

Figure 1. Baseline angiography shows long and diffuse stenosis of the right coronary artery (RCA) involving its aortic ostium (A). Angiography of the RCA after Schockwave balloon modification (B). Final angiographic result after stent implantation (3.0 $\times 44 \mathrm{~mm}$ distally and $3.5 \times 18 \mathrm{~mm}$ proximally) and non-compliant balloons post-dilatation $(3.25 \times 12 \mathrm{~mm}$ distally and $4.0 \times 12 \mathrm{~mm}$ proximally at $24 \mathrm{~atm})(\mathrm{C})$. The intravascular ultrasound (IVUS) images: baseline (D1, E1, F1), after shockwave balloon modification (D2, E2, F2) and final result (D3, E3, F3). Small white lines in Figure 1A point to placement of IVUS images on the RCA angiography, respectively D, E and F. Small white stars $\left({ }^{*}\right)$ in the Figure 1D2-F2 show cracks in calcified plaques with concomitant acoustic shadowings; MLA - minimal lumen area. 